\title{
An interactive environment for digital registration of human behaviour for biofeedback purposes
}

\author{
Tomasz Szymczyk ${ }^{1, *}$, and Katarzyna Baran $^{1}$ \\ ${ }^{1}$ Lublin University of Technology, Poland
}

\begin{abstract}
Unceasing development of computing power of modern computers and advanced graphics card functions enable very faithful projections of the virtual world. However, issues related to real-time object generation and object interaction are complex research problems. A designer and programmer face a dilemma of modifying the model. Simplifying the model significantly affects minimisation of a detail. The article presents a position of digital registration of human behaviour in the virtual world. A study of human behaviour has been proposed, where the main stimulant is not an image but a sound. Participants heard different sounds and responded to them. Parameters such as: pulse, hand movement, general posture and movements of the entire body were registered. An analysis of change in the scope of given psychological modality is important for biofeedback, which allows activating a self-regulatory ability by a body's feedback. In the authors' research, depending on variability of a respondent's reaction, a further interaction with the virtual sound object is created. In this environment, fear of dogs was safely examined. The paper presents the results of pilot studies, which indicated that it is possible to control human reactions by analysing registered parameters, learning to control their own reactions and with more frequent biofeedback training - working out concentration and coping in the most extreme conditions.
\end{abstract}

\section{Introduction}

IT tools have been facilitating biofeedback for many years. This is particularly important in the treatment of anxiety and phobias that impede normal functioning in everyday life. The development of appropriate human self-regulatory abilities through feedback allows for minimisation and even elimination of fears. It is important for implementation of new technologies and technical means to register physiological modalities to be safe and correct.

This article focused on the possibility of registering human behaviour in the virtual world and using results for biofeedback purposes. Current attitude of researchers in the use of virtual reality for research on anxiety and emotions was presented by literature review. Next, the authors presented research. In the virtual environment with a stimulant in the form of sound, the fear of dogs was examined. The results of pilot studies indicated that it is possible to control and manage their own reactions. It is assumed that with more frequent biofeedback training, there would be achieved a decrease in anxiety, increased focus and better coping with even an extreme anxiety situation. The authors in this study did not treat people, only proposed and tested an interactive environment.

\subsection{Specificity and psychological aspects of phobias}

Phobia as neurotic disorders manifest themselves in persistent fear of specific situations, phenomena or objects whose appearance makes it difficult to function. Often, however, these things are not dangerous. The fear in phobia is so overwhelming that it is often not controlled. The causes of the phobia may be traumas and psychological injuries created in childhood or in adulthood, psychodynamic mechanisms (projection, transfer of aggression) or even genes. Characteristic symptoms of phobias are: fast heartbeats, shaking hands, shortness of breath, high sweating, lacrimation, blood pressure disorders, and body numbness. Methods for phobias treatment are different [1], but the first way is exposure to anxiety. So, a therapist brings a situation where a patient has to deal directly with his phobia. $\mathrm{He}$ does not have a chance to escape or avoid confrontation. The results of the confrontation are compared with the previously created anxiety analysis. The therapist assesses the scale of anxiety and special features. Other treatments are: desensitisation (based on relaxation training) [2], implosive therapy (contact with phobia several times within a short time span) [3], modelling (implementation of correct behaviour to life) [4], pharmacological medicines.

The most common types of phobias are the following five [5]: animals - mostly insects, reptiles and amphibians; the natural environment - water, storm; blood and injections; situations - fear of flying, elevator or public communication, other phobias - avoiding choking, infection.

*Corresponding author: t.szymczyk@pollub.pl 


\subsection{Biofeedback to treat phobias}

Biofeedback as a method of providing man with feedback about his physiological changes is applicable not only in psychology, medicine but also in sport and business [6]. When treating phobia, it is crucial to understand the object, situation or other anxiety factor and learn to deliberately modify the body's functions for normal functioning. The anxiety factor (stimulant) can be both, the whole surrounding virtual environment (for example: glass bridge, dark basement), as well as individual virtual objects (spider, snake, hornet, skull, etc.) The process of creating the virtual environment is described in [7] and modelling of small individual objects in [8]. Great attention is paid to an ability to concentrate and master. Supporting phobia treatment with modern technologies facilitates registration of parameters, their thorough analysis and reference in biofeedback [9]. The results of measurements are an important element for psychotherapy because they allow evaluating the effectiveness of therapy and improving the results during further exercises based on previous results [10]. Consultation with a psychologist or therapist and choosing the right therapy are an opportunity to minimise the phobia and even eliminate it. Developing the right patterns of behaviour, improving awareness of the threat and the level of anxiety are the main steps in the treatment of phobias.

\section{Literature review}

Along with technological development, there is a noticeable trend of introducing interdisciplinary research and applications of technologies. This review focuses on the indication of the use of virtual reality in the phobia study. The practical application of VR technology was already the subject of interest of scientists at the end of the 20th century [11]. The first mention of the use of VR as an effective method of treating mental disorders can be found in many articles [2], [9], [12]. Jose P. Monge and others [13] presented the VR Phobia system. He allowed his patients to be placed in a virtual world in which they could learn proper techniques of reacting to anxiety triggers (desensitisation method). The therapy was based on visual and auditory interaction. The created prototype was consulted with cognitive psychology therapists and was used as a tool in the therapy process.

The reduction of phobia against spiders using virtual reality was done by Per Calbring [14]. He conducted a controlled clinical trial comparing the effectiveness of standard OST (One Session Therapy) therapy and a onetime VRET (Virtual Reality Exposure Therapy) gaming session based on VR equipment. 98 subjects with spider phobia were divided into two groups (OST and VRET) and tested at 1-week intervals. Each study ended with a behavioural test. The results showed that VRET is an effective alternative to OST or it can be regarded as an enhancement after OST.

The system of perception of the real world with audiovisual stimulation in the treatment of phobias was proposed by Jessica S. Ortiz and others [15]. The system consists of several different virtual environments suited to the phobia being treated.
Therapy is determined by a psychotherapist. During one biometric signals are recorded, then processed and a control algorithm is created. The patient can control his behaviour by seeing the signals graphically and being stimulated by virtual and audiovisual stimuli.

The potential treatment of social phobias by their triggering with three-dimensional images is presented in [16]. 21 patients (10 women and 11 men) with social phobia participated in 12 image exposure sessions, each 50 minutes long. The average number of sessions was seven. As a result of the research, there was an improvement in patients' condition and reduced social anxiety ( 6 months after the end of treatment). The need to conduct further research confirming the findings of the authors was considered.

Simulations of real interactive experiments are presented in [17]. The authors, through a literature review, pointed to effectiveness of VR in therapy for specific phobias and post-traumatic stress disorder. They also drew attention to limitations and possible directions of further development. Research related to the treatment of phobias with the use of VR can also be found in [18], [19].

\section{Research - materials and methods}

\subsection{Research procedure}

The research concerned the possibility of using the interactive environment to stimulate the fear of kynophobia along with biofeedback.

Due to the research ethics and test phase of research, authors focused only on a study of kynophobia, where the level of anxiety has reached the value of $5-$ the greatest fear. The pilot study involved three people aged 24-38 who declared fear of dogs (kynophobia).

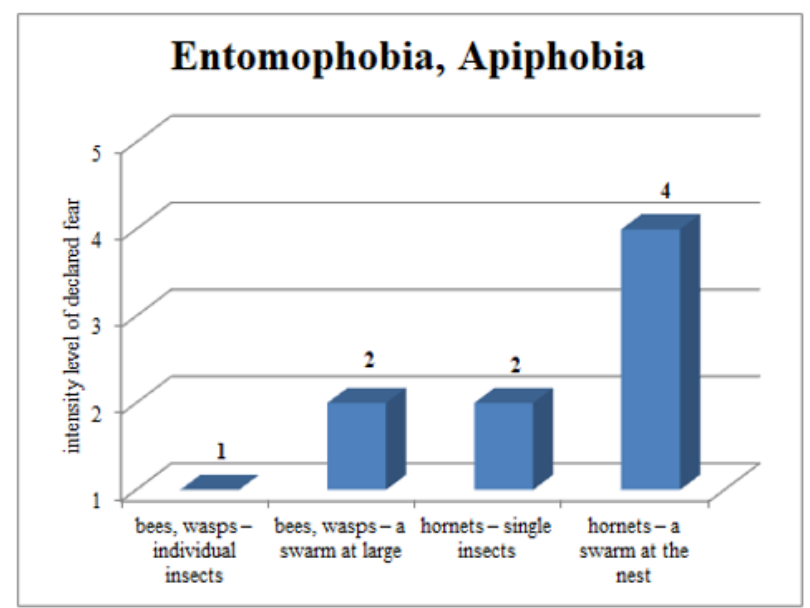

Fig. 1. Survey results for entomophobia and apiphobia (median value).

The investigative procedure was conducted in two stages. Stage 1 was a survey carried out among 44 people ( 3 women and 41 men) aged 24-40 (median 25 years). Respondents rated on a scale of 1-5 (1-none, 5very intensely) as they are afraid of certain anxiety states. The results are divided into groups of phobias and shown in Figures 1-4. 


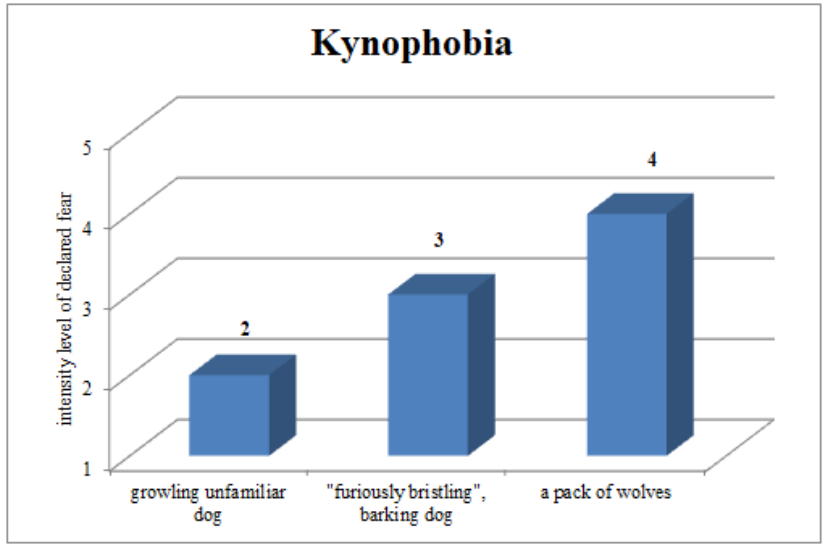

Fig. 2. Survey results for kynophobia (median value).

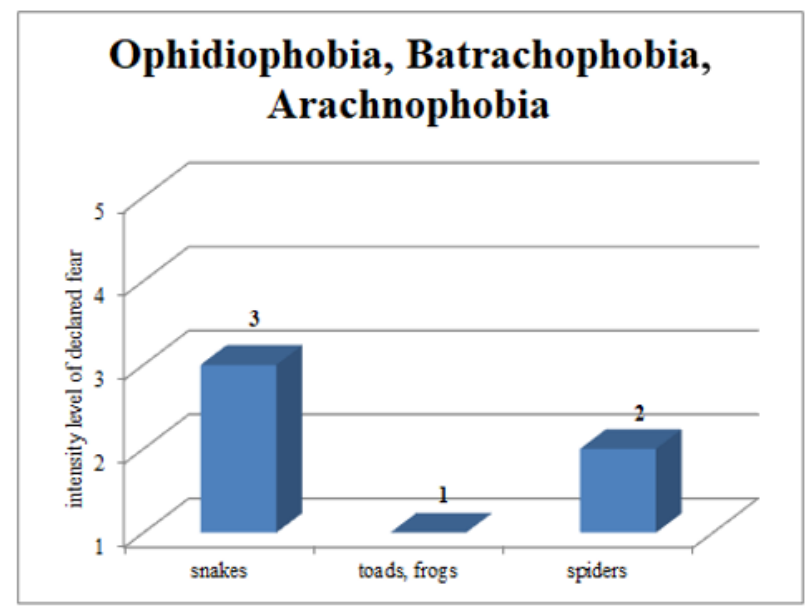

Fig. 3. Survey results for ophidiophobia, batrachophobia, arachnophobia (median value).

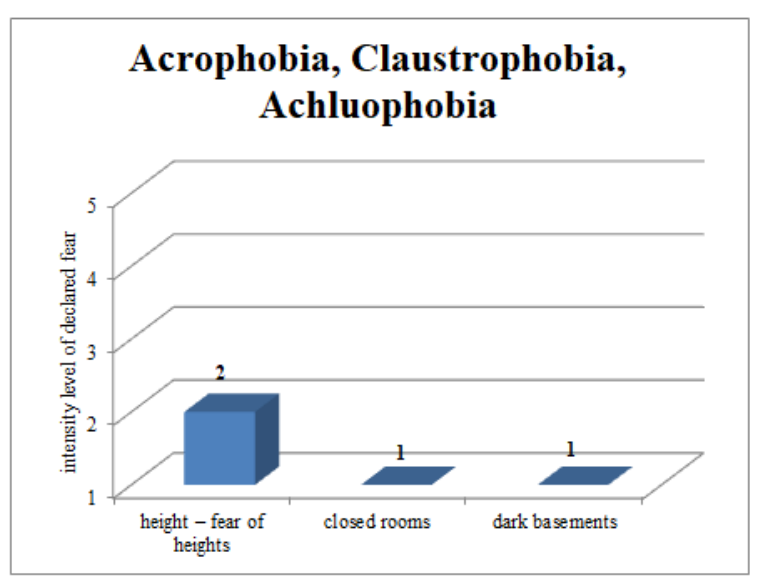

Fig. 4. Survey results for acrophobia, claustrophobia, achluophobia (median value).

Obtained results of the questionnaire confirmed that students are afraid of certain objects less and more of the others. There is a strong variation in both types and intensity of anxiety. With such differentiation, it is very difficult to identify unreliable (untruthful) survey responses. For the purpose of at least elementary verification, a reference question about the fear of a wolf pack was introduced. Exactly every adult person should show fear of this animal. All 44 people rated this fear highly (median value 4 Figure 2). Panic fear was declared by $14 \%$ (6 people) as shown in Figure 5.

To the second stage of the research, people who declared fear of dogs at level 5 were invited. They were 3 people out of 6 (from 14\%) who confirmed panic and agreed to participate in further research.

\section{The percentage of people declared panic fear of dogs}

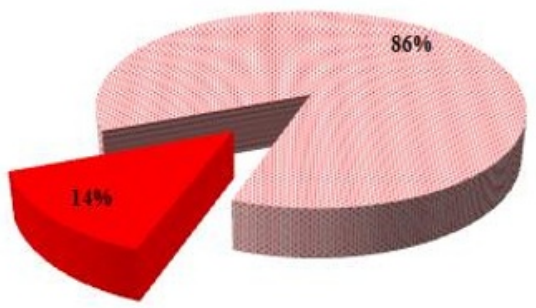

- The number of people who are afraid of dogs (panicly). "11 The others

Fig. 5. Declaration of kynophobia among the respondents.

Stage II - the main research procedure - consisted of the following stages:

- Interview about the subject's disease and its immediate family members (epilepsy, heart disease, hypertension, etc.).

- Classification of a person to be tested.

- Familiarisation and signing declarations and documents required by the Ethics Committee.

- Launching testing software, applying gloves, a heart rate monitor, VR glasses covering the eyes.

- Relaxation time - turning on relaxation music, determination of resting heart rate, measurement of skin moisture, body temperature and measurements of humidity and ambient temperature.

- Sound Projection - approx. 3-5 minutes session, finished accordingly to the decision of the respondent.

- Re-relaxation time - taking a sitting position by the subject. Repeated measurements.

The stimulus of fear was sound. Samples of running sounds, dog's barking - three types of sound were used:

- Level I: clear "animal's running”,

- Level II: growling and gentle barking of a dog,

- Level III: furious barking of a dog.

The sound was fully interactive and coupled with the tested user. Using advanced software, it was possible to move the sound source closer or further to the recipient, thanks to which he had an impression of a real presence of a dangerous dog.

In the study two scenarios were proposed. The first one used VR glasses, in which the subject could see a very dark forest with a very clearly moving dark object Figure 6a. The fact that it was impossible to identify the object, stimulated imagination and intensified an element of uncertainty and fear. The second way was to cover the eyes and stimulate through the sound - Figure $6 \mathrm{~b}$. 


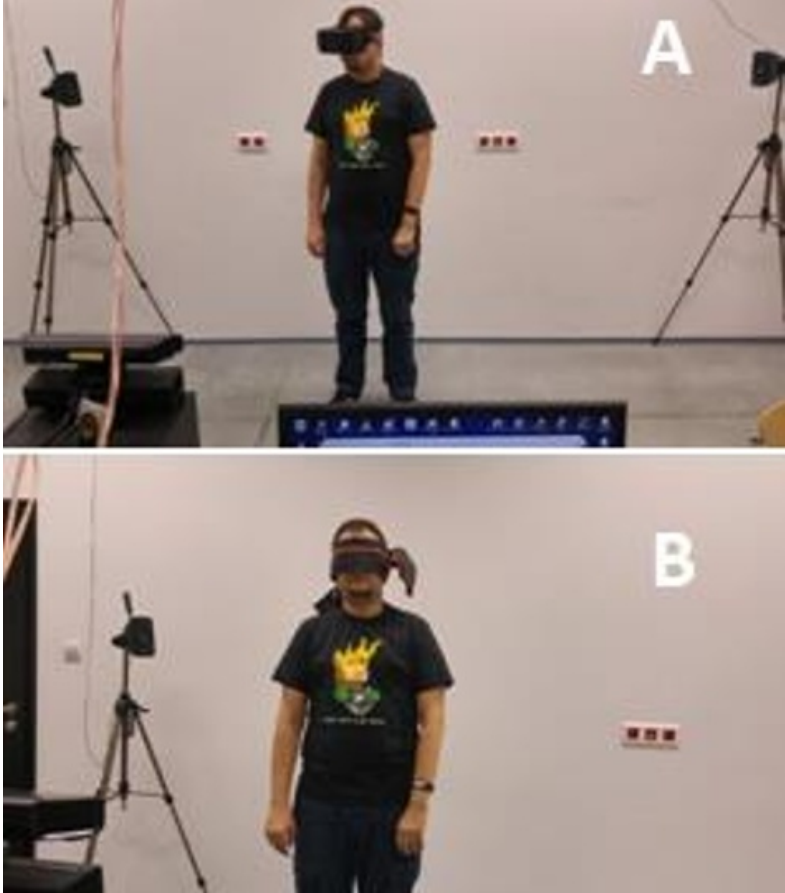

Fig. 6. The examined person according to scenario I (VR glasses) - part A and scenario II (blind covering of eyes) - part B.

Figure 7 and Figure 8 show views from the virtual environment. Figure 7 presents dark forest in brightened version and original, which was seen by examined person. Figure 8 is a view of the object approaching the moving subject.

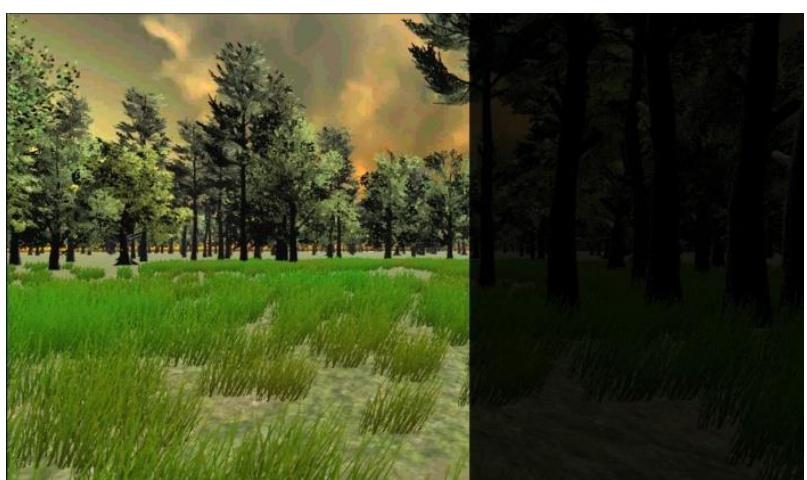

Fig. 7. View of the dark forest - on the left the picture is brightened, on the right - the original one.

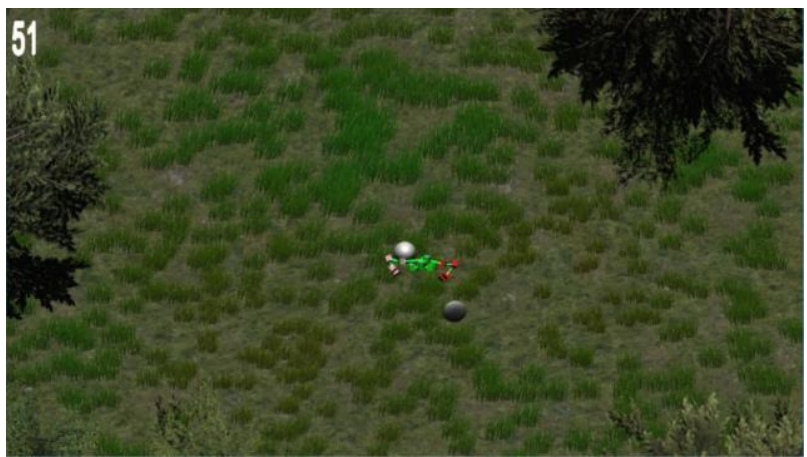

Fig. 8. View of the object approaching the moving subject (the pulse of the subject was indicated in the upper left corner-51).
Figure 9 demonstrates an example of the distancing of the virtual danger at the end of movement of the subject and simultaneously after the training that reduces the pulse.

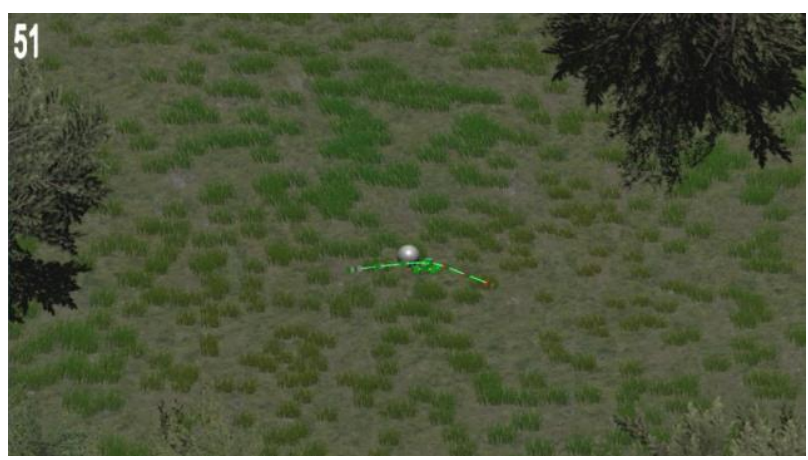

Fig. 9. View of the object moving away from the still standing test subject (in the upper left corner, the subject's pulse was indicated - 51).

\subsection{Description of the test bench}

The research stand is an additional module in the already existing position of studying human behaviours stimulated by virtual reality.

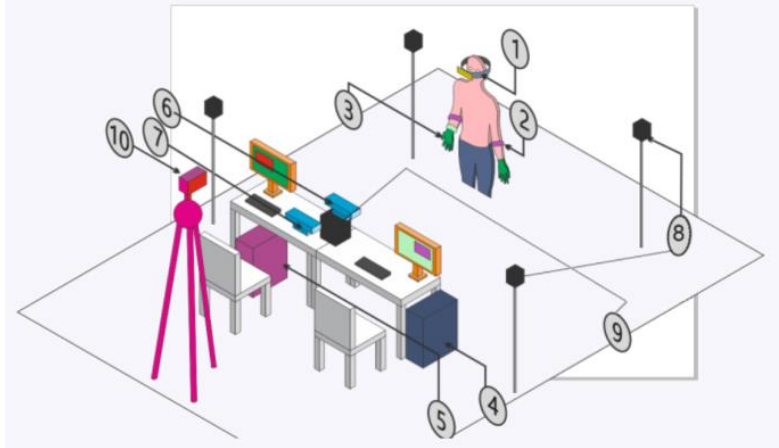

Fig. 10. Visualisation of a structure of the behaviour study stand (division into component elements).

The module for sound projection of phobias consists of the following elements - Figure 10:

- VR glasses (resolution 960x1080 px per eye, refresh rate $75 \mathrm{~Hz})-1$.

- Hand pulse sensor, which task is to interact with the world of VR and save the history of the pulse -2 .

- Contraction of fingers recorded through fibre optic gloves (minimum sampling rate $75 \mathrm{~Hz}$, one sensor per finger, sensor resolution 12-bit) -3 .

- Computer set for VR image generation (octa-core processor, 48 GB RAM, graphic card: memory interface 256-bit, $1600 \mathrm{GHz}, 8 \mathrm{~GB}$ memory) - 4 .

- Computer set for recording data from the motion recorder -5 .

- Motion sensing input devices, which task is to interact with the Virtual Reality scene -6 .

- Digital motion recorder - its task is to record data of movement of the person's profile -7 . 
- 3D surround sound set. Its task is to generate realistic spatial sound and focus it on a given, variable distance and direction in relation to the examined person -8 .

- Subwoofer - a woofer generating low, powerful bass which directly and acts on the senses of the subject 9.

$-4 \mathrm{~K}$ reference camera used for registration of the whole research. Thanks to the recordings accumulated on it, it is possible, in case of ambiguity, to later interpret measurement data -10

In addition, the station uses a digital thermometer to study the body temperature of the respondent, a thermometer with a hygrometer to measure temperature and humidity in the room. The digital skin moisture meter (skin detector) was also used.

\section{Discussion of results}

The fear of dogs in the survey was the highest classified by the respondents. Research with the use of VR threat projection requires a huge power of computing the computer graphics card. The problem is to generate an interactive, dangerous, natural-looking dog. Natural interactive animation, the use of the technique of "hairs" is not only a challenge for the graphics card but also for the graphics and the designer. Any simplification of the aggressive dog model makes it simply grotesque, funny and completely destroys the results of research on such a facility. The authors, therefore, proposed interaction only with sound or very visible object. The conducted research and the literature review presented above confirms the huge potential of VR use in the field of anxiety research. A common practice of collaborating researchers with psychologists was also recognized. This is necessary due to medical and ethical aspects, the safety of the subject, and the size of the subject in general. The authors also plan to establish lasting cooperation with psychologists.

Figure 11 presents a fragment of the pulse record of the subject. There are numerous disturbances in area A. It is connected with the establishment of the heart rate meter as well as the general excitement accompanying the examination process.

In area $\mathrm{B}$, relax music is projected and a so-called resting pulse is obtained. This pulse will later be the reference level for interactive sound presentation.

Area $\mathrm{C}$ is a part of the movement study, when a person turns around, jumps up, in a word "provokes" a virtual threat.

High physical activity that causes an ever stronger, more serious auditory stimulus translates unambiguously into an increase in the pulse of the subject. There is also a very large diversity of pulse values in this phase (high pulse dynamics). The pulse variation interval (blue range) assumed by the authors is definitely wider than in phase B.

The last phase D is an attempt to lower the heartbeat in order to move the virtual object away from itself. The area in Figure 11, painted in blue, is the correct area, while the red line indicates the real values obtained during the measurement for one person.
In phase $\mathrm{D}$, two stages can clearly be distinguished. The first is an attempt to calm down and thus reduce the heartbeat. In the absence of sudden movements, lowering of the pulse and "virtual fears" - the goal is approaching. The goal is to gain peace - the value of a pulse similar to those of the relaxation phase (phase B). Apart from the errors of the test method and recording devices, it is clearly visible that the heartbeat is reduced, which is within the given tolerance.

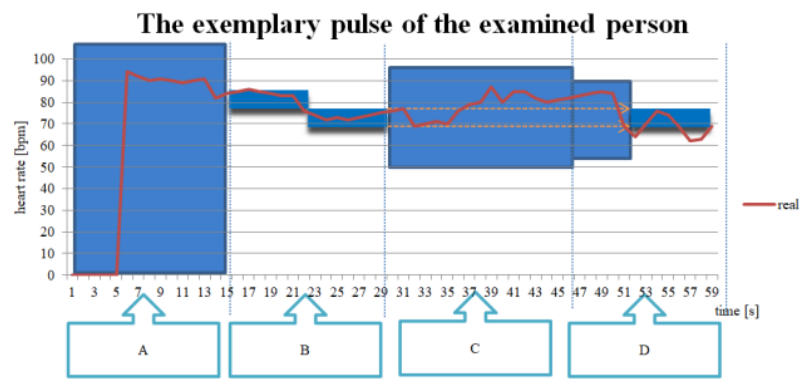

Fig. 11. Fragment of an example pulse.

After this phase, the study and projection of relaxation music came to the end. However, this is not already demonstrated in this graph.

Pilot studies have proved that there is a possibility to train reactions that cause "calming down", both body and pulse movements. This gives grounds for stating that the position presented in the future may become a training position in the process of stopping kynophobia.

\section{Conclusion}

An interactive environment for digital registration of human behaviour is safe for the respondent. It facilitates simulation of real situations with the possibility of their quick termination in case of too much emotional or anxious escalation of the subject.

Pilot studies have shown that individual studies allow assessing the level of anxiety and indicating at what stage the fear of dogs is the greatest.

The authors claim that conducting a training session (several test tests within a few weeks), would prove that regular exercise and analysis of own reactions allows to reduce anxiety, control initial fear, develop focus and preserve the rationality of thinking even in situations originally considered extreme or limiting. It would also be important to consult a psychologist or a therapist about the results.

The research program titled "Study of human behaviour induced by visual stimuli originating from the virtual reality environment", performed in the Laboratory of Intelligent Systems Programming and Computer 3D Technology 'Lab 3D' was approved by the Commission for Research Ethics, No. 7/2016 dated 19.12.2016

\section{References}

1. P. E. Nathan, J. M. Gorman, A guide to treatments that work (Fourth Edition, Oxford 2015)

2. P. J. Lang, Foundations of Behavioral TherapyeBook (Routledge, New York, 2017) 
3. T. G. S. Stampfl, Foundations of Behavioral Therapy-eBook (Routledge, New York, 2017)

4. I. Milosevic, R. E. McCabe, Phobias: The Psychology of Irrational Fear (Greenwood, Oxford, 2015)

5. P. Muris, Handbook of Childhood Psychopathology and Development Disabilities Treatment (Springer, Cham, 2017)

6. K. Baran, T. Szymczyk, EDULEARN 2018, ISSN:2340-1117, 8806-8813 (2018)

7. T. Szymczyk, INTED 2018, ISSN 2340-1079, 176185 (2018)

8. T. Szymczyk, INTED 2018, ISSN 2340-1079, 164169 (2018)

9. D. Reyna, R. Caraza, M. Gonzalez-Knoell, A. Ayala, P. Martinez, A. Loredo, R. Rosas, P. Reyes, Springer 213 (2018)

10. P. M. Lehrer, Int. J. of Psych.,Elsevier 131(2017)

11. W. Greenleaf, IEEE Eng. in Med. and Bio. Mag. 15, 23-30 (1996)
12. C. Eichenberg, C. Wolters, Intech 35-64 (2012)

13. J. P.Monge, G. Lopez, L. A. Guerrero, Ad. in Int. Sys. and Com., Springer 482 (2017)

14. P. Carlbring, (San Francisco,2017)

15. J. S. Ortiz, P. M. Velasco, W. X. Quevedo, M. Alvarez, J. S. Sanchez, P. Carvajal, Lec. Not. in Com. Sci.,Springer 10325 (2017)

16. C. M. Gebara, T. P. de Barros-Neto, L. Gertsenchtein, F. Lotufo-Neto, Rev. Bras. de Psiq. 38 (1) (2016)

17. J. L. Maples-Keller, C. Yasinski, N. Manjin, B. O. Rothbaum, Neur. 14 (3), 554-563, (2017)

18. C. Botella, J. Fernandez-Alvarez, V. Guillen, A. Garcia-Palacios, R. Banos, Cur. Psych. Rep. 19 (7) (2017)

19. D. Freeman, S. Reeve, A. Robinson, A. Ehlers, D. Clark, B. Spanlang, M. Slater, Psych. Med. 47 (14) (2017). 